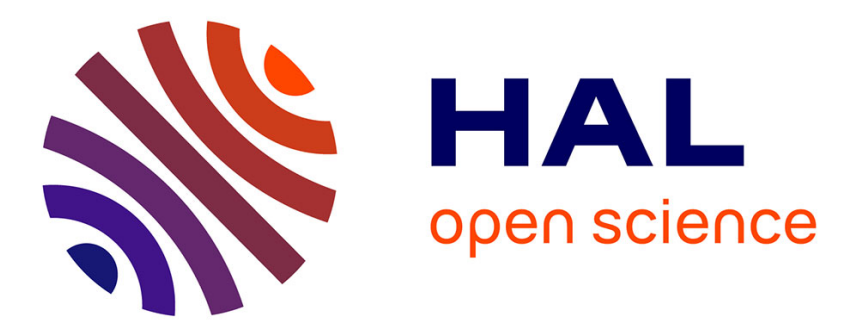

\title{
Cross conversion between surface plasmon polaritons and quasicylindrical waves
}

X. Y. Yang, Haitao Liu, Philippe Lalanne

\section{To cite this version:}

X. Y. Yang, Haitao Liu, Philippe Lalanne. Cross conversion between surface plasmon polaritons and quasicylindrical waves. Physical Review Letters, 2009, 102 (15), pp.153903. 10.1103/PhysRevLett.102.153903 . hal-00570586

\section{HAL Id: hal-00570586 \\ https://hal-iogs.archives-ouvertes.fr/hal-00570586}

Submitted on 31 Mar 2016

HAL is a multi-disciplinary open access archive for the deposit and dissemination of scientific research documents, whether they are published or not. The documents may come from teaching and research institutions in France or abroad, or from public or private research centers.
L'archive ouverte pluridisciplinaire HAL, est destinée au dépôt et à la diffusion de documents scientifiques de niveau recherche, publiés ou non, émanant des établissements d'enseignement et de recherche français ou étrangers, des laboratoires publics ou privés. 


\title{
Cross Conversion between Surface Plasmon Polaritons and Quasicylindrical Waves
}

\author{
X. Y. Yang, ${ }^{1,2}$ H. T. Liu, ${ }^{1,3}$ and P. Lalanne ${ }^{1}$ \\ ${ }^{1}$ Laboratoire Charles Fabry de l'Institut d'Optique, CNRS, Univ Paris-Sud, Campus Polytechnique, \\ 91127 Palaiseau cedex, France \\ ${ }^{2}$ School of Optoelectronics Science and Engineering, Huazhong University of Science and Technology, \\ Wuhan 430074, People's Republic of China \\ ${ }^{3}$ Key Laboratory of Opto-electronic Information Science and Technology, Ministry of Education, Institute of Modern Optics, \\ Nankai University, Tianjin 300071, People's Republic of China \\ (Received 27 September 2008; published 14 April 2009)
}

\begin{abstract}
The optical properties of textured metallic surfaces are governed by the scattering of surface plasmon polaritons (SPPs) and of quasicylindrical waves (CWs), which are both excited by the nano-objects located on the surface. We study here a fundamental scattering process of these fields, namely, the cross conversion of a CW into a SPP. We first show that this inelastic process is inevitable in multi-nanoobject ensembles and then propose a procedure enabling a rigorous calculation of the cross conversion scattering coefficients. Additionally, by mapping this intricate process to a much simpler one, we derive general and simple expressions for the cross conversion efficiency. All predictions are carefully supported by fully vectorial computational results.
\end{abstract}

DOI: 10.1103/PhysRevLett.102.153903

PACS numbers: 42.25.Fx, 42.79.Ag, 73.20.Mf, 78.66.-w

Metallic surfaces that are nanotextured play a central role in the emerging field of plasmonics [1]. At a microscopic level, their macroscopic (collective) optical properties are governed by two distinct elementary waves that are excited by individual nano-objects and then propagate on the surface, before eventually interacting with nearby nano-objects: the well-known surface plasmon polariton (SPP), i.e., a surface mode [1], and a residual quasicylindrical wave $(\mathrm{CW})$, i.e., an electromagnetic field with radiative and evanescent components that persists along the surface over a few-wavelength propagation distance [2-5]. For noble metals, both waves are equally excited at visible frequencies, but at longer wavelengths, the $\mathrm{CW}$ rapidly becomes the preponderant field $[2,6]$. The SPP excitation or scattering by individual or arrayed nano-objects has been studied extensively and is well comprehended nowadays [1,7-10]. Just as SPPs, CWs participate in the energy transfer between the nano-objects, but unfortunately, virtually nothing is presently known on their scattering properties [2-6]. In this Letter, we remove this deficiency and provide the first study of a fundamental $\mathrm{CW}$-scattering process, namely, the inelastic scattering of CWs into SPPs. We show that this process virtually exists for all metallic textured surfaces, we evidence that it impacts the optical property of the surfaces especially at visible frequencies, and we further discuss how its efficiency scales with the energy of the light.

Because SPPs are modes of the metal interface, their scattering coefficients obey reciprocity relationships and can be calculated through normal mode concepts $[9,10]$. CWs are not modes (they are a superposition of evanescent and propagation fields), and the analysis of their scattering coefficient is much more intricate conceptually. We start with a proof of existence for the cross conversion phenomenon by numerically studying the SPP-generation rate of a groove doublet on a metal surface. Thanks to a simplified coupled-wave model, we derive a simple procedure to rigorously calculate the cross conversion coefficients. We further map the inelastic problem into a much simpler one, which involves only purely elastic SPP scatterings. This allows us to derive general expressions for the cross conversion coefficient and to directly apply all the available knowledge (including the theoretical and numerical tools) on SPPs to the inelastic scattering problem.

CW-to-SPP conversion is inherent to multiple scattering and can be virtually observed whenever a set of subwavelength particles is arrayed on a metal surface. To evaluate the importance of the cross conversion, we consider the simplest system that sustains such a conversion, namely, a groove doublet on a metallic surface [see Fig. 1(a)]. Figure 1(b) shows the scattered magnetic field for a normally incident plane wave $(\lambda=974 \mathrm{~nm})$ polarized along the $x$ axis. The fully vectorial numerical calculations are performed with an aperiodic Fourier modal method [11] (a-FMM), using the frequency dependent gold permittivities $\varepsilon_{g}$ tabulated in [12]. Hereafter, we consider the field scattered on the right side of the doublet on the surface $(z=0)$, see Fig. 1(c). The total field (solid-red curve) is composed of a SPP (dashed-blue) and of a CW (dottedblack), which are codirectionally launched out. The SPP contribution is extracted from the total field by using the orthogonality between the SPP mode and the radiative modes, and by difference, the $\mathrm{CW}$ contribution is obtained $[2,10]$. The decomposition allows us to calculate a SPPexcitation efficiency, defined as the fraction of the incident power, which is directly impinging onto the groove aper- 

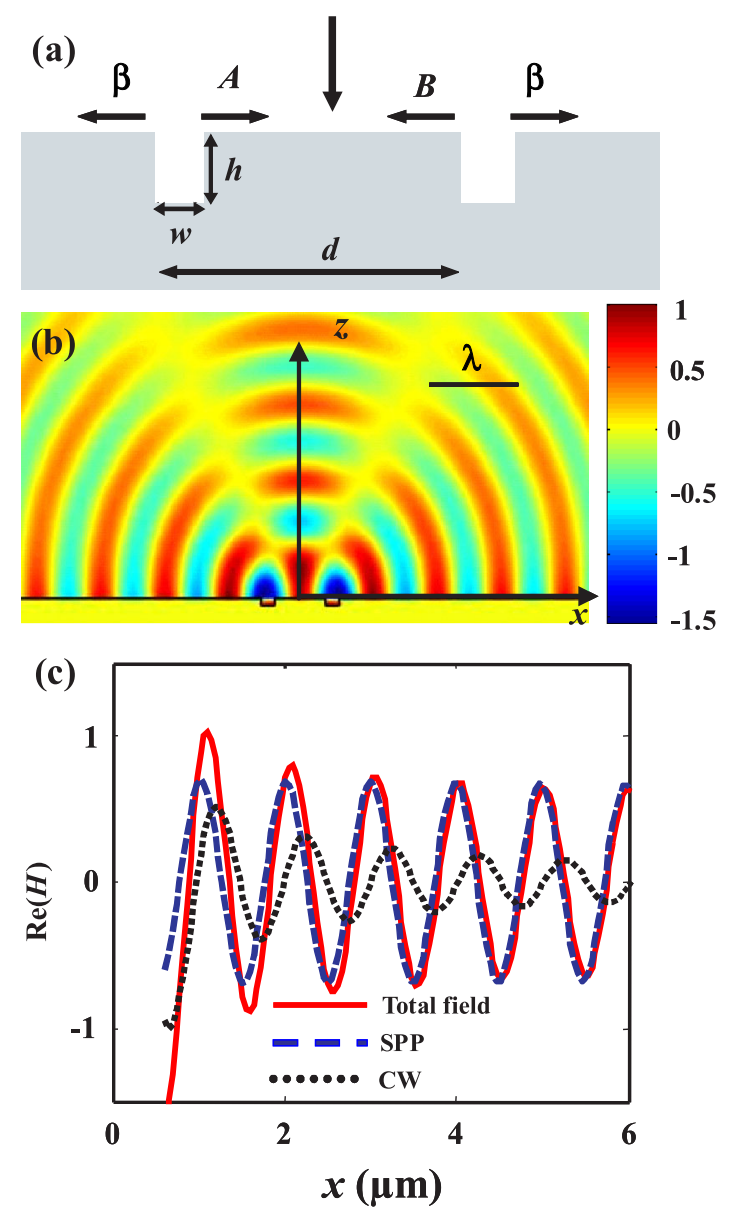

FIG. 1 (color online). Electromagnetic field scattered by a groove doublet under illumination at normal incidence by a plane wave polarized along the $x$ axis. (a) Schematic of the groove-doublet configuration, including the geometrical parameters $w, h$, and $d$, and the electromagnetic quantities $A, B$, and $\beta$ used in the model. (b) Scattered magnetic field $\operatorname{Re}(H)$. The incident and specularly reflected plane waves have been removed for the sake of clarity. (c) Magnetic field scattered on the air-gold surface $(z=0)$ on the right side of the doublet. The total field (solid-red) is composed of a SPP mode (dashed-blue) and of a CW (dotted-black). The calculations are performed for $h=$ $0.1 \lambda, w=0.2 \lambda$ and $d=\lambda\left(\lambda=974 \mathrm{~nm}, \quad \varepsilon_{g}=-44.02+\right.$ 3.24i).

tures and which is converted into the two SPPs launched on both sides of the doublet. Hereafter the efficiency will be normalized by that associated to a single groove. Using the a-FMM, we have repeatedly calculated the efficiency by varying the groove separation distance $d$ in the interval $w / 2<d<4 \mu \mathrm{m}$.

The normalized SPP-generation efficiencies $\eta$ are shown in Fig. 2 with black-solid curves for two groove depths, $h=0.1 \lambda$ and $0.2 \lambda$. They exhibit an oscillatory fringe pattern with a high contrast. The pattern can be essentially understood as resulting from the interference between the two SPPs generated by the two grooves.

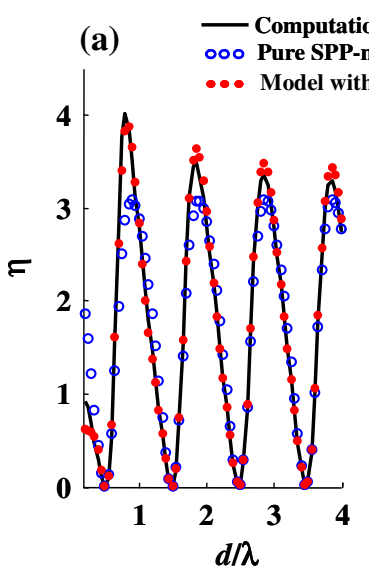

(b)

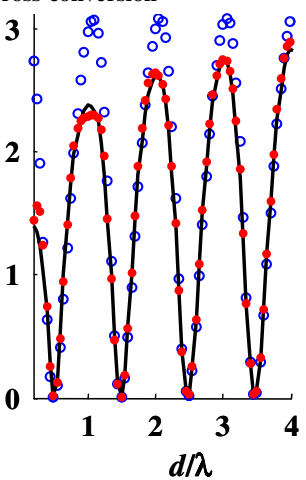

FIG. 2 (color online). Normalized SPP-generation efficiency $\eta$ as a function of the groove separation distance. A quantitative agreement is achieved between the a-FMM data (solid-black) and the model predictions of Eq. (4) (red dots), while the pure SPP model (blue circles) fails at predicting the transient regime. The results are obtained for $w=0.2 \lambda(\lambda=974 \mathrm{~nm})$ and for $h=0.1 \lambda$ (a) and $h=0.2 \lambda$ (b). For $d>4 \mu \mathrm{m}$, other calculations show that the oscillation amplitude remains essentially constant.

However, as will be shown hereafter, this pure SPP description [shown with the blue circles in Figs. 2(a) and 2(b)] cannot predict the transient regime observed for small $d$ 's. Before reaching a stationary behavior for $d>4 \mu \mathrm{m}$ (not shown), the maxima of $\eta$ present an important falloff in Fig. 2(a) or riseup in Fig. 2(b). The transient is a direct signature of CW-to-SPP cross conversions occurring at the grooves, and as will be shown hereafter, quantitative information on the amplitude and on the phase of the cross conversion coefficients can be gained by analyzing the fine details of the fringe pattern.

To explore this question, we next develop a coupledwave model that takes into account the individual CWs generated by each groove and their further conversions into SPPs by scattering at the other groove. But before, let us consider the magnetic field $H(x)$ scattered on the surface by a single groove illuminated by a normally incident plane wave. This field (Fig. B in [2]) can be accurately described as a combination of a SPP and of a CW,

$$
H(x)=\beta_{\mathrm{SP}} H_{0} \exp \left(i k_{\mathrm{SP}} x\right)+\beta_{\mathrm{CW}} H_{0}(x / \lambda)^{-m} \exp \left(i k_{0} x\right),
$$

where $k_{\mathrm{SP}}=k_{0}\left[\varepsilon_{g} /\left(\varepsilon_{g}+1\right)\right]^{1 / 2}$ is the complex SPP propagation constant, $k_{0}=2 \pi / \lambda, m \approx 0.5$ is the attenuation exponent of the $\mathrm{CW}$, and $H_{0}$ is a positive normalization constant [2]. Equation (1) defines two complex scattering coefficients, $\beta_{\mathrm{SP}}$ and $\beta_{\mathrm{CW}}$, which represent the relative weights of the SPP and the CW contributions to the total field at a single-wavelength distance from the groove.

With this notation in mind, we now derive a set of coupled-wave equations for the groove doublet. Let us 
denote by $A$ and $B$ the unknown SPP modal coefficients scattered in the positive and negative $x$ directions [Fig. 1(a)]. Neglecting high-order cross conversion processes, we have

$$
A=\beta_{\mathrm{SP}}+r_{\mathrm{SP}} B u+r_{c} \beta_{\mathrm{CW}} v .
$$

$A$ results from three contributions, a direct excitation by the left-handed groove under the plane-wave illumination, an elastic SPP back reflection at the same groove, and an inelastic backscattering of the $\mathrm{CW}$ initially originated by the illumination of the right-handed groove. Similarly for the generation coefficient $\beta$ of the SPP launched on the right side of the doublet [see Fig. 1(a)], we may write

$$
B=\beta_{\mathrm{SP}}+t_{\mathrm{SP}} A u+t_{c} \beta_{\mathrm{CW}} v .
$$

In Eqs. (2) and (3), $u=\exp \left(i k_{\mathrm{SP}} d\right)$ and $v=(d / \lambda)^{-m} \times$ $\exp \left(i k_{0} d\right)$ are the SPP and CW phase delays, $r_{\mathrm{SP}}$ and $t_{\mathrm{SP}}$ are the SPP elastic reflection and transmission coefficients at a groove, and $r_{c}$ and $t_{c}$ are the unknown cross conversion coefficients associated to the inelastic scattering of an incident $\mathrm{CW}$ into reflected and transmitted SPP modes. Indeed, as will be confirmed hereafter, $r_{c}$ and $t_{c}$ (like the elastic coefficients $r_{\mathrm{SP}}$ and $t_{\mathrm{SP}}$ ) may depend on the groove parameters, $h$ or $w$, but are independent of $d$. At normal incidence, $B=A$ and from Eqs. (2) and (3), one obtains

$$
\beta=\beta_{\mathrm{SP}}\left(1+\frac{u t_{\mathrm{SP}}}{1-u r_{\mathrm{SP}}}\right)+\beta_{\mathrm{CW}} v\left(t_{c}+r_{c} \frac{u t_{\mathrm{SP}}}{1-u r_{\mathrm{SP}}}\right) .
$$

As expected, the normalized SPP efficiency given by $\eta=$ $|\beta|^{2} /\left|\beta_{\mathrm{SP}}\right|^{2}$ results from two contributions. The first one (the first term on the right side of the equation) corresponds to a contribution that solely depends on SPP modes (earlier called the pure SPP model), and the second one is due to the additional $\mathrm{CW}$ cross conversion process under analysis. The two contributions may sum up constructively [Fig. 2(a)] or destructively [Fig. 2(b)] for different groove parameters since the quantities in Eq. (4) (except $u$ and $v$ ) depend on the groove parameters.

To extract the inelastic scattering coefficient, we adopt a two-step procedure. We first calculate the elastic scattering coefficients $r_{\mathrm{SP}}$ and $t_{\mathrm{SP}}$ with the a-FMM. This step does not pose any problem since many numerical tools (like ours) may provide these modal scattering coefficients. In a second step, using Eq. (4), we fit the calculated $\beta$ values over the full interval of separation-distances $(w / 2<d<$ $4 \mu \mathrm{m})$ by optimizing the unknown complex parameters $r_{c}$ and $t_{c}$. As shown in Fig. 2, the model well captures the initial transient zone and a quantitative agreement is obtained between the calculated data (black curve) and the fitted curve (red dots) evaluated for the optimized parameters.

The two-step procedure provides a rigorous numerical approach to calculate the cross conversion coefficients. In principle, by repeatedly using it for different nanoparticle geometries, one should be able to study the impact of various physical parameters on the cross conversion efficiency. However, although viable this would be burdensome and, more importantly, this would not be helpful for understanding why some particles may offer efficient cross conversion and some others not. As we shall see, it is possible to gain much more insight by mapping the cross conversion coefficient (including their phase) into those of a much simpler problem.

For that let us consider two elementary scattering problems at the same frequency. In the first problem, a SPP is impinging onto a subwavelength object (a groove or a ridge for instance). The second problem corresponds to the scattering of a CW by the same object. Although they are very different by nature (the SPP is a mode, the CW is not, the damping characteristics are different, and so on), the $\mathrm{CW}$ and SPP fields at the metal surface $(z=0)$ share many properties (they have nearly identical propagation constants, similar penetration depth in the metal, and so on), and in addition, they may have identical fields on the object under adequate normalization. Referring to a form of causality principle where equal causes have equal effects [13], one expects that the scattered fields of the two diffraction problems are nearly identical: same scatterings into free space, same excitations of the groove FabryPerot resonances and in the context of the cross conversion, one should expect that the SPPs contained in the two scattered fields be the same. Formally, we write

$$
r_{c} \approx r_{\mathrm{SP}}, \quad t_{c} \approx t_{\mathrm{SP}}-1 .
$$

These equations are remarkably simple. Their validity has been thoroughly verified for different particle geometries (grooves and ridges) over a broad spectral range, from the visible to the near-infrared. The results obtained for grooves with $w=0.2 \lambda$ and $h=0.1 \lambda$ are summarized in Figs. 3(a) and 3(b). The $r_{c}$ and $t_{c}$ coefficients (plusses) are obtained by applying the rigorous double-step procedure at every wavelength $\lambda$, while the $r_{\mathrm{SP}}$ and $t_{\mathrm{SP}}$ coefficients (black-solid curves) are straightforwardly calculated using the a-FMM. As we vary the wavelength, we also scale all geometrical dimensions. Clearly, Eq. (5) is quantitatively verified, even for situations relying on very strong scatterings at visible wavelengths.

Because they bridge an intricate inelastic scattering problem to a much simpler elastic one, Eqs. (5) are meaningful. They are conceptually interesting since they attach scattering coefficients to waves that are not normal modes. They are useful since they considerably ease the calculation of cross conversion strengths, by replacing the intricate double-step procedure by direct calculations of SPP scattering coefficients. Finally, they are also intuitively helpful. For example, they provide important scaling behaviors, such as the frequency dependence [14] of the cross conversion coefficients, which is not a priori intuitively clear. Thanks to Eq. (5), one may instead think in terms of a 

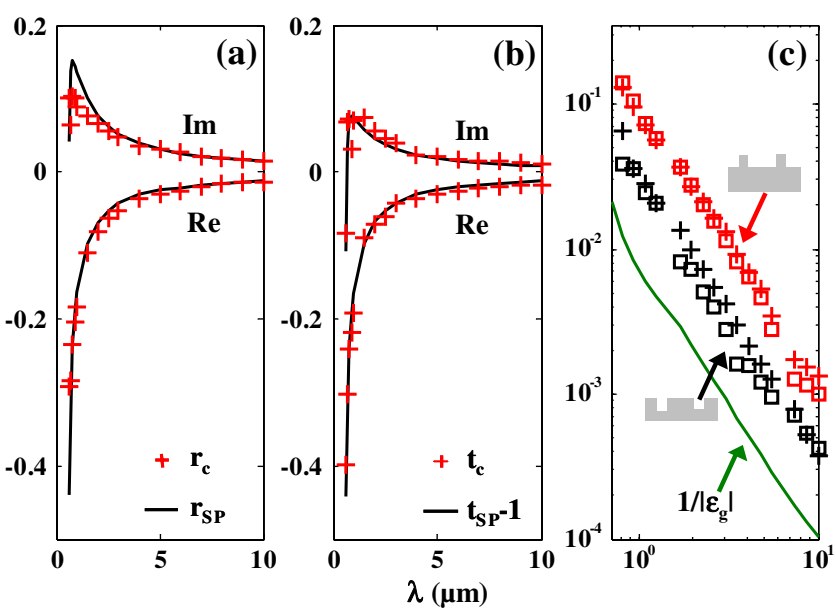

FIG. 3 (color online). Wavelength dependence of the cross conversion scattering coefficients $r_{c}$ and $t_{c}$. (a) and (b) Test of Eq. (5) for grooves $(w=0.2 \lambda$ and $h=0.1 \lambda)$. The plots compare the cross conversion coefficients, $r_{c}$ and $t_{c}$ (red plusses), obtained with the double-step procedure and the elastic scattering SPP coefficients, $r_{\mathrm{SP}}$ and $t_{\mathrm{SP}}$ (solid curves), calculated with the aFMM. (c) The cross conversion efficiencies $\left|r_{c}\right|^{2}$ (plusses) and $\left|t_{c}\right|^{2}$ (squares) scale as the inverse of the metal permittivity $\left|\varepsilon_{g}\right|^{-1}$ shown with the green solid curve. The black (respectively red) marks are obtained for groove (respectively ridge) doublets using the double-step procedure. The ridge widths and heights are $0.2 \lambda$ and $0.1 \lambda$, respectively.

pure elastic scattering problem, and by noting that the electromagnetic field of a normalized SPP mode on the interface scales as $\left|\varepsilon_{g}\right|^{-1 / 4}$, one easily infers that the cross conversion efficiencies $\left|r_{c}\right|^{2}$ and $\left|t_{c}\right|^{2}$ both scale as $\left|\varepsilon_{g}\right|^{-1}$. This scaling law has been quantitatively checked numerically by comparing the wavelength dependence of the metal permittivity (solid-green curve) with the efficiencies deduced from the double-step procedure (plusses and squares), see Fig. 3(c). The comparison is performed for groove (black marks) and ridge (red marks) doublets, showing that the scaling law is a general property that is not restricted to specific particle geometries. Additionally, it is noteworthy that the $\left|\varepsilon_{g}\right|^{-1}$ dependence for the cross conversion efficiency largely differs from those associated to the CW and SPP-generation efficiencies, $\left|\beta_{\mathrm{CW}}\right|^{2}$ and $\left|\beta_{\mathrm{SP}}\right|^{2}$, which, respectively, vary as $\left|\varepsilon_{g}\right|^{1 / 2}$ and $\left|\varepsilon_{g}\right|^{-1 / 2}$ due to the distinct excitation behaviors of the two waves $[2,10]$.

In conclusion, we have theoretically studied a new process on metal films, namely, the cross conversion of a CW into a SPP mode. We have provided a direct proof of existence, derived general simple expressions of the cross conversion efficiencies, and shown that these efficiencies strongly depend on the metal conductivity. We believe that the cross conversion is a fundamental process that impacts the optical property of many (if not all) textured metallic surfaces. For large and periodic particle ensembles, in contrast to the doublets studied here, multiple cross conversions occur and strong constructive or destructive interference can be anticipated. Since our current understanding of the electromagnetic properties of textured metallic surfaces relies on microscopic models that largely ignore the CWs [6], we expect that this analysis will be helpful for ultimately understanding the dynamics and behavior of plasmonic systems.

Haitao Liu acknowledges the "Fondation FrancoChinoise pour la Science et ses Applications" (FFCSA) and the China Scholarship Council (CSC). Xiaoyan Yang acknowledges the CSC. The authors thank Jean Paul Hugonin for fruitful discussions.

[1] C. Genet and T. W. Ebbesen, Nature (London) 445, 39 (2007).

[2] P. Lalanne and J. P. Hugonin, Nature Phys. 2, 551 (2006); see EPAPS Document No. E-PRLTAO-102-068917 for supplementary material. For more information on EPAPS, see http://www.aip.org/pubservs/epaps.html.

[3] L. Chen, J. T. Robinson and M. Lipson, Opt. Express 14, 12629 (2006).

[4] G. Lévêque, O. J. F. Martin, and J. Weiner, Phys. Rev. B 76, 155418 (2007)

[5] L. Aigouy et al., Phys. Rev. Lett. 98, 153902 (2007).

[6] H. T. Liu and P. Lalanne, Nature (London) 452, 728 (2008).

[7] G. Gay et al., Phys. Rev. Lett. 96, 213901 (2006).

[8] W. L. Barnes et al., Phys. Rev. B 54, 6227 (1996); J. Seidel et al., Appl. Phys. Lett. 82, 1368 (2003); H. F. Schouten et al., Phys. Rev. Lett. 94, 053901 (2005); H. W. Kihm et al., Appl. Phys. Lett. 92, 051115 (2008).

[9] J. A. Sanchez-Gil and A. A. Maradudin, Appl. Phys. Lett. 86, 251106 (2005); A. Y. Nikitin and L. Martin-Moreno, Phys. Rev. B 75, 081405(R) (2007).

[10] H. T. Liu et al., IEEE J. Sel. Top. Quantum Electron. 14, 1522 (2008).

[11] J. P. Hugonin and P. Lalanne, J. Opt. Soc. Am. A 22, 1844 (2005).

[12] E. D. Palik, Handbook of Optical Constants of Solids (Academic Press, NY, 1985).

[13] Within the Green's function formalism, this can be concluded from the integral equation satisfied by scattered fields; see, for instance, A. B. Evlyukhin et al., Phys. Rev. B 76, 075426 (2007).

[14] More specifically, the frequency dependence of $\left|r_{c}\right|^{2}$ and $\left|t_{c}\right|^{2}$ is solely due to the metal dispersion, since for dispersionless materials, Maxwell's equations are invariant. 